\title{
Drone Positioning for User Coverage Maximization
}

\author{
Jingcong Sun, and Christos Masouros \\ Dept. of Electronic and Electrical Engineering, University College London, London, U.K. \\ Email:\{uceejsu,c.masouros\}@ucl.ac.uk
}

\begin{abstract}
Aerial base stations (BSs) based on unmanned aerial vehicles (UAVs) can provide rapid wireless services to users in areas without ground infrastructure. This paper aims to deploy multiple aerial BSs to cover a maximum number of ground users within a certain target area while avoiding inter-cell interference (ICI). Two techniques are proposed. The first method deploys multiple aerial BSs in a successive way and converts the non-convex constraints into various linear constraints which can be easily solved. The second method simultaneously deploys multiple aerial BSs by dividing the target area into $K$ convex subareas with the help of $K$-means clustering. Simulation results show that both techniques achieve a performance gain compared to the benchmark circle packing theory (CPT).
\end{abstract}

\section{INTRODUCTION}

Aerial base stations (BSs) based on rotary-wing Unmanned aerial vehicles (UAVs) have been increasingly appealing to wireless communication systems for providing rapid wireless services to ground users. UAVs which are cost-effective, flexible and likely to have a higher probability of line-of-sight (LoS) channels are suitable for various related scenarios [1]. For example, UAVs can be deployed to offer fast service recovery or supply when fixed communication infrastructures are out-of-service. However, the practical use of aerial BSs is hindered by several challenges, among which the efficient placement of UAVs that can cover a maximum number of ground users is of paramount importance.

The work in [2] found the optimal altitude to place aerial BSs which can maximize the coverage area on the ground. Instead of just maximizing the coverage area, algorithms aiming at covering the maximum number of users have been increasingly focused on [3]-[5]. Specifically, a 3-D circle placement problem is solved in [3] by formulating it as a mixed integer non-linear problem (MINLP), while [4] made a further step by taking various quality-of-service (QoS) constraints into consideration. However, none of the above mentioned works considered the case of using multiple aerial BSs which limits their use. In most real situations, however, the use of a single aerial BS can hardly meet the requirement of most ground users. Situations of deploying two UAVs have been considered by Authors in [6]. Although circle packing theory (CPT) is utilized in [7] to maximize the total coverage area of multiple aerial BSs, specific user distributions are not considered in the work. More recently, a spiral algorithm proposed in [8] achieves a $100 \%$ user coverage probability. However, the study introduces interference issue, which needs to be tackled with overlaid techniques.

The aim of this paper is studying the efficient placement of multiple aerial BSs in order to obtain a maximum user coverage probability while completely avoiding the influence of inter-cell interference (ICI). Following [7], [8], we assume that the locations of ground users are known with the help of high-precision GPS systems and each aerial BS has enough capacity to offer services to all the users it covers. We consider a scenario where multiple UAVs are deployed in the lack of ground BSs' coverage. The aerial BS placement problem is modelled as a circle placement problem and no coverage overlap is allowed so that ICI between aerial BSs is intrinsically avoided. We propose a successive deployment method based on linear approximation and a simultaneous deployment technique with the help of $K$-means clustering to solve the placement problem. Our simulation results demonstrate that the proposed circle placement methods achieve higher user coverage probability than the benchmark CPT [7].

\section{SYSTEM MODEL}

Consider a square target area with side length $L_{s}$ containing a set of low-mobility users denoted by $\mathcal{M}$. We assume $K$ multiple aerial BSs are deployed within the region in order to provide wireless communication to as many ground users as possible. Note that, as UAVs can move freely, such deployment of aerial BSs can be done regularly in order to accommodate any changes in the user positions. In this paper, we only focus on each snapshot of users within the area instead of studying the flying trajectory of UAVs.

Among multiple models characterizing air-toground(AtG) links, we utilize the most general one proposed in [2] which considers both the effect of LoS links and non-line-of-sight (NLoS) links. If we denote the location of user $i$ in the set $\mathcal{M}$ as $\left(x_{i}, y_{i}\right)$, the horizontal location and the altitude of the $k$-th UAV as $\left(x_{c k}, y_{c k}\right)$ and $h_{k}, k=1,2, \ldots K$ respectively, then the ground distance between user $i$ and UAV $k$ is $r_{i k}=\sqrt{\left(x_{i}-x_{c k}\right)^{2}+\left(y_{i}-y_{c k}\right)^{2}}$. Accordingly, the probability of having a LoS link is:

$$
P_{\mathrm{LoS}}=\frac{1}{1+a \exp \left(-b\left(\frac{180}{\pi} \theta_{i k}-a\right)\right)}
$$


where $a$ and $b$ are parameters depending on the environment and $\theta_{i k}=\tan ^{-1}\left(\frac{h_{k}}{r_{i k}}\right)$ is the elevation angle in radians. Note that the probability of having an NLoS link is represented by $P_{\mathrm{NLOS}}=1-P_{\mathrm{LoS}}$.

As shown in [2], path loss of a specific propagation group can be modeled as free space path loss plus an excessive path loss which depends on the propagation type. The weighted path loss between user $i$ and the $k$ th aerial BS, which is a function of $\theta_{i k}$ and $r_{i k}$ is then expressed as

$$
\begin{array}{r}
\mathrm{PL}\left(\theta_{i k}, r_{i k}\right)=\frac{A}{1+a \exp \left(-b\left(\frac{180}{\pi} \theta_{i k}-a\right)\right)}+ \\
20 \log \left(\frac{r_{i k}}{\cos \left(\theta_{i k}\right)}\right)+B
\end{array}
$$

where $A=\eta_{\mathrm{LoS}}-\eta_{\mathrm{NLoS}}$ and $B=20 \log \left(\frac{4 \pi}{c}\right)+$ $20 \log \left(f_{c}\right)+\eta_{\mathrm{NLoS}}$ are constants. $f_{c}$ is the carrier frequency of the system, $c$ is the light speed, $\eta_{\mathrm{LoS}}$ and $\eta_{\mathrm{NLoS}}$ denote the excessive loss for LoS and NLoS links respectively.

In this paper, the service threshold is defined in terms of the path loss. To be specific, user $i$ is covered by the $k$-th aerial $\mathrm{BS}$ when the propagation path loss is smaller than or equal to a threshold value $\gamma_{t h}$. It is clear that the coverage area of each aerial BS is a circle area with radius $R_{k}$, where $R_{k}=\left.r_{i k}\right|_{\mathrm{PL}\left(h_{k}, r_{i k}\right)=\gamma_{t h}}$. It has been shown in [3] that, the maximum coverage radius is always associated with a fixed elevation angle $\theta_{\mathrm{opt}}$, which only depends on the environment. Therefore, the threshold path loss can be written as

$$
\begin{array}{r}
\gamma_{t h}=\frac{A}{1+a \exp \left(-b\left(\frac{180}{\pi} \theta_{\text {opt }}-a\right)\right)}+ \\
20 \log \left(\frac{R_{k}^{*}}{\cos \left(\theta_{\text {opt }}\right)}\right)+B
\end{array}
$$

from which we can solve $R_{k}^{*}$, which is the maximum radius we can obtain, when a specific $\gamma_{t h}$ is given. Note that when multiple UAVs are deployed, the interference effect needs to be addressed. It is clear that ICI can be intrinsically avoided when there is no overlap between coverage areas of aerial BSs.

\section{DEPLOYMENT METHOD WITH LINEAR APPROXIMATION (LA)}

In this section, a method based on successive circle placement to find the optimal locations of aerial BSs such that a maximum number of ground users are covered is proposed. Following [7], [8], we assume that all UAVs have the same radius $R$ and are thus deployed in the same altitude $H$ when a specific path loss requirement is given, that is

$$
\begin{aligned}
& R_{k}=R, k=1,2, \ldots, K \\
& h_{k}=H=R \tan \left(\theta_{\text {opt }}\right), k=1,2, \ldots, K
\end{aligned}
$$

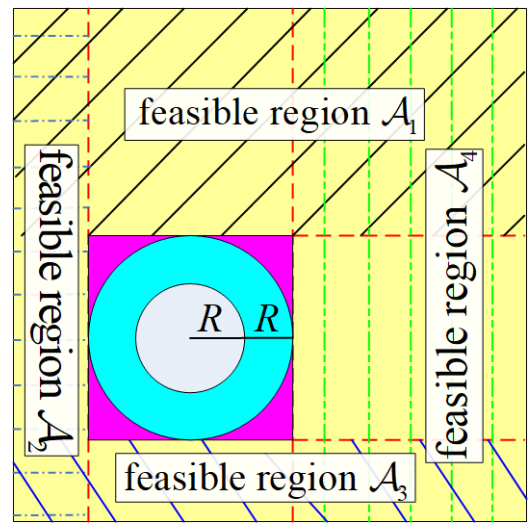

Fig. 1. Converting the non-convex feasible region into four convex feasible regions with linear approximation

with $R$ calculated from (3). Therefore, the problem boils down to a circle placement problem that we want to position multiple circles in the horizontal plane such that the number of enclosed user points is maximized. UAVs are placed in a successive method, where at each step the placement of the new aerial BS aims to cover the maximum number of remaining users in the target area while ensuring that there is no overlap between coverage areas. The location of the first UAV can be found by utilizing the method proposed in [3]. Let Boolean variable $u_{i} \in\{0,1\}, i \in \mathcal{M}$ denote the status of user $i$ such that the user is enclosed by the first aerial BS when $u_{i}=1$ and is not covered when $u_{i}=0$. Then the circle placement problem is formulated as

$$
\begin{aligned}
& \underset{x_{c 1}, y_{c 1}, u_{i}}{\operatorname{maximize}} \sum_{i \in \mathcal{M}} u_{i} \\
& \text { subject to } \\
& \left(x_{i}-x_{c 1}\right)^{2}+\left(y_{i}-y_{c 1}\right)^{2} \leq R^{2}+M\left(1-u_{i}\right) \\
& u_{i} \in\{0,1\}, \forall i \in \mathcal{M}
\end{aligned}
$$

where $\left(x_{c 1}, y_{c 1}\right)$ is the horizontal location of the first $\mathrm{UAV}$, and $M$ is a very large constant.

However, when we want to position the second UAV, an additional constraint guaranteeing no overlap between coverage areas is needed. To ensure this, the distance between the centers of the two aerial BSs in the horizontal plane should be no smaller than $2 R$. Correspondingly, the placement of the second UAV is formulated as

$$
\begin{aligned}
& \underset{x_{c 2}, y_{c 2}, u_{i}}{\operatorname{maximize}} \sum_{i \in \mathcal{M}} u_{i} \\
& \text { subject to } \\
& \left(x_{i}-x_{c 2}\right)^{2}+\left(y_{i}-y_{c 2}\right)^{2} \leq R^{2}+M\left(1-u_{i}\right) \\
& \left(x_{c 2}-x_{c 1}\right)^{2}+\left(y_{c 2}-y_{c 1}\right)^{2} \geq 4 R^{2} \\
& u_{i} \in\{0,1\}, \forall i \in \mathcal{M}
\end{aligned}
$$

where $\left(x_{c 2}, y_{c 2}\right)$ is the horizontal location of the second UAV. Unfortunately, the additional constraint is nonconvex which makes (6) unsolvable. 
In Fig. 1, the circle in white with radius $R$ represents the coverage area of the first aerial $\mathrm{BS}$, and the region which is not covered by the blue circle with radius $2 R$ is the feasible region which satisfies the non-convex constraint in (6) to place the center of the second UAV. We notice that such a non-convex region which specifies all the feasible locations of the second UAV in the horizontal dimension can be approximated into four linear regions which are convex, if we relax the blue circular region into a square region such that the original blue circle is surrounded by the purple square as shown in Fig. 1. Therefore, instead of solving (6), we can solve four MINLP problems with different linear constraints, each has the following form

$$
\operatorname{maximize}_{x_{c 2}, y_{c 2}, u_{i}} \sum_{i \in \mathcal{M}} u_{i}
$$

subject to

$$
\begin{aligned}
& \left(x_{i}-x_{c 2}\right)^{2}+\left(y_{i}-y_{c 2}\right)^{2} \leq R^{2}+M\left(1-u_{i}\right) \\
& y_{c 2} \geq y_{c 1}+2 R, \text { if }\left(x_{c 2}, y_{c 2}\right) \in \mathcal{A}_{1} \\
& x_{c 2} \leq x_{c 1}-2 R, \text { if }\left(x_{c 2}, y_{c 2}\right) \in \mathcal{A}_{2} \\
& y_{c 2} \leq y_{c 1}-2 R, \text { if }\left(x_{c 2}, y_{c 2}\right) \in \mathcal{A}_{3} \\
& x_{c 2} \geq x_{c 1}+2 R, \text { if }\left(x_{c 2}, y_{c 2}\right) \in \mathcal{A}_{4} \\
& u_{i} \in\{0,1\}, \forall i \in \mathcal{M}
\end{aligned}
$$

where $\mathcal{A}_{1}, \mathcal{A}_{2}, \mathcal{A}_{3}$ and $\mathcal{A}_{4}$ are the four feasible regions which are convex as shown in Fig. 1. The optimal horizontal location of the second UAV as well as the maximum number of enclosed ground users are then found by solving four MINLP problems. Note that the effective area for placing the second UAV is reduced by $(16-4 \pi) R^{2}$ as a result of relaxing the infeasible circle region into a new square area.

The optimization problem of placing the $k$-th UAV $(k>1)$ is formulated as

$$
\begin{aligned}
& \underset{x_{c k}, y_{c k}, u_{i}}{\operatorname{maximize}} \sum_{i \in \mathcal{M}} u_{i} \\
& \text { subject to } \\
& \left(x_{i}-x_{c k}\right)^{2}+\left(y_{i}-y_{c k}\right)^{2} \leq R^{2}+M\left(1-u_{i}\right) \\
& \left(x_{c k}-x_{c j}\right)^{2}+\left(y_{c k}-y_{c j}\right)^{2} \geq 4 R^{2} \\
& u_{i} \in\{0,1\}, \forall i \in \mathcal{M}, j=1,2, \ldots, k-1
\end{aligned}
$$

where $\left(x_{c k}, y_{c k}\right)$ and $\left(x_{c j}, y_{c j}\right)$ denote the location of the $k$-th UAV and the $j$-th UAV in the horizontal dimension respectively. We first convert each of the $k-1$ non-convex constraints into four convex constraints as illustrated above. Note that the four convex regions of each of the $k-1$ previously deployed aerial BSs only specify the areas avoiding ICI between the $k$-th and that specific aerial BS. In order to find the feasible regions which guarantee the coverage area of the $k$-th UAV does not overlap with the coverage area of any previously deployed aerial BSs, we first find all possible intersections of $(k-1)$ convex areas. To be specific,

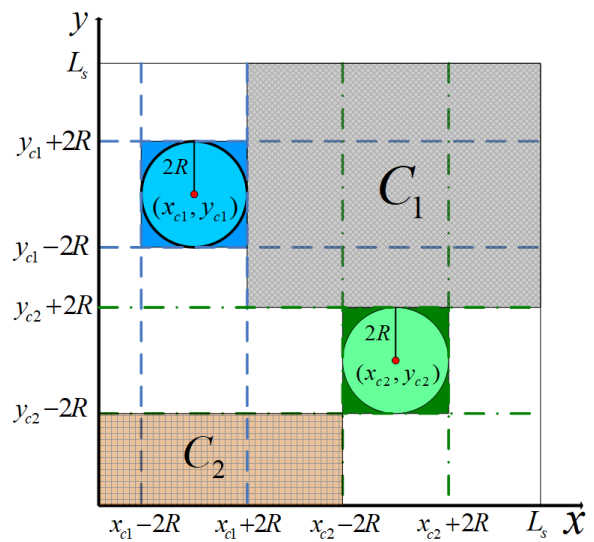

Fig. 2. An example of obtaining feasible regions for placing the third aerial BS

for each of the $k-1 \mathrm{UAVs}$, we choose one of the four generated convex regions and use logic function to find the intersection of these $k-1$ selected regions. For placing the $k$-th UAV, a total of $4^{k-1}$ intersections should thus be generated and each intersection is denoted as $C_{z}, z=1,2, \ldots, 4^{k-1}$. After obtaining all the $4^{k-1}$ intersections, an elimination method is utilized to find all feasible regions. All sets which are null sets or sets which turn out to be subsets of other generated intersections are eliminated, and the remaining sets are the feasible regions we should search for.

Fig. 2 shows an example of obtaining feasible regions for positioning the third UAV. If we denote the horizontal location of the third UAV as $\left(x_{c 3}, y_{c 3}\right), C_{1}$ : $\left\{x_{c 3}, y_{c 3} \mid x_{c 1}+2 R \leq x_{c 3} \leq L_{s}, y_{c 2}+2 R \leq y_{c 3} \leq L_{s}\right\}$ is one of the feasible regions we should search for. The region is generated by taking the intersection of $\quad\left\{x_{c 3}, y_{c 3} \mid x_{c 1}+2 R \leq x_{c 3} \leq L_{s}, 0 \leq y_{c 3} \leq L_{s}\right\}$, which is one of the convex regions of the first UAV, and $\quad\left\{x_{c 3}, y_{c 3} \mid 0 \leq x_{c 3} \leq L_{s}, y_{c 2}+2 R \leq y_{c 3} \leq L_{s}\right\}$, which is one of the four convex regions associated with the second aerial BS. However, the region $C_{2}$ : $\left\{x_{c 3}, y_{c 3} \mid 0 \leq x_{c 3} \leq x_{c 2}-2 R, 0 \leq y_{c 3} \leq y_{c 2}-2 R\right\}$ turns out to be a subset of another generated intersection $\left\{x_{c 3}, y_{c 3} \mid 0 \leq x_{c 3} \leq L_{s}, 0 \leq y_{c 3} \leq y_{c 2}-2 R\right\}$, and is thus eliminated. The total number of feasible regions of the $k$-th UAV is denoted as $N_{M}^{k}$. In this case, instead of solving (8), we can solve $N_{M}^{k}$ MINLP problems, each with the following form

$$
\operatorname{maximize}_{x_{c k}^{m}, y_{c k}^{m}, u_{i}} \sum_{i \in \mathcal{M}} u_{i}
$$

subject to

$$
\begin{aligned}
& \left(x_{i}-x_{c k}^{m}\right)^{2}+\left(y_{i}-y_{c k}^{m}\right)^{2} \leq R^{2}+M\left(1-u_{i}\right) \\
& \left(x_{c k}^{m}, y_{c k}^{m}\right) \in C_{k}^{m} \\
& u_{i} \in\{0,1\}, \forall i \in \mathcal{M}
\end{aligned}
$$

where $C_{k}^{m}$ is the $m$-th feasible region of the $k$-th aerial BS, $\left(x_{c k}^{m}, y_{c k}^{m}\right)$ is the optimal location of the $k$-th UAV 
in region $C_{k}^{m}, m=1,2, \ldots, N_{M}^{k}$. If we denote the number of covered users by solving the $m$-th optimization problem as $N_{m}$, and denote the maximum $N_{m}$ for all $m$ as $N_{\max }$, we have $\left(x_{c k}, y_{c k}\right)=\left.\left(x_{c k}^{m}, y_{c k}^{m}\right)\right|_{N_{m}=N_{\max }}$.

\section{DePloyment METHOD BASED ON CLUSTERING} (CL)

The above proposed algorithm introduces exponentially increasing computational complexity due to the need to solve $4^{k-1}$ logic combination operations, which makes its use prohibitively complex when a large number of aerial BSs is needed. In this section, a method which simultaneously deploys multiple UAVs with the help of $K$-means clustering is proposed. The method avoids ICI and can be used for target areas of any size.

$K$-means clustering classifies points into $K$ clusters by iteratively computing the center of clusters which is represented by the mean value of points within each Voronoi cell and reassigning each point into updated cluster centers until the position of all cluster centers keep unchanged [9]. In our particular scenario, we notice that the whole target area can be divided into $K$ subareas with boundary lines forming the Voronoi diagram, by assigning user points into $K$ clusters. The intelligent division of the target area brings great benefit to the deployment of multiple UAVs in several senses. First, each subarea which is bounded by few straight lines or line segments is a convex region, within which we can solve an optimization problem similarly to (5) to find the maximum number of enclosed user points. In addition, The boundary lines of Voronoi diagram ensure that the aerial BSs placed in each subarea will not overlap with each other, so ICI is intrinsically avoided. Furthermore, a number of $K$ UAVs can be deployed simultaneously, so we avoid the latency and dependence on previously deployed aerial BSs as using LA.

After partitioning the user points into $K$ clusters and, subsequently, dividing the whole target area into $K$ subareas, we first need to find the largest allowed coverage area within each subarea to avoid ICI. As the shape of each subarea is a polygon, it is likely that certain subareas can only accommodate circles with radii smaller than $R$. Assume the $k$-th subarea is formed with $\mathrm{N}_{k l}$ line segments or straight lines, each line is expressed in the form of $y=a_{k l} x+b_{k l}, l=1,2, \ldots, \mathrm{N}_{k l}$, where $a_{k l}$ and $b_{k l}$ represents the slope and offset of the line respectively. It is known that for any point $\left(x_{d}, y_{d}\right)$, if $y_{d}-a_{k l} x_{d}-b_{k l}<0$, the point is in the region below the line. On the contrary, if $y_{d}-a_{k l} x_{d}-b_{k l}>0$, the point is in the region above the line.

We note that the boundary lines of each subarea also implicate a feasible region for placing the circle center. To be specific, the distance between the circle center and each boundary line should be no smaller than the length of radius of the circle. Therefore, if we denote the maximum allowed radius of the circle placed in the

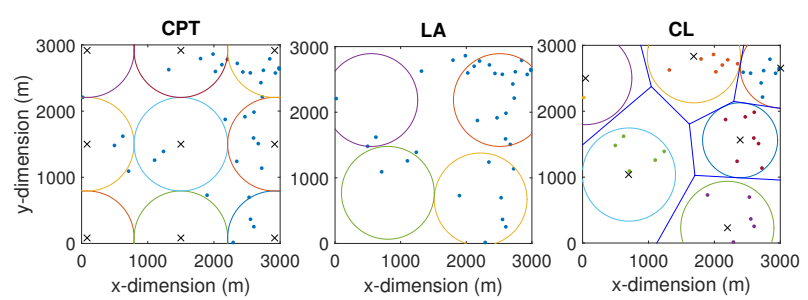

Fig. 3. Example of placing multiple aerial BSs

$k$-th subarea as $R_{\text {max }}^{k}$, the radius of the $k$-th circle is then $R_{k}=\min \left(R, R_{\max }^{k}\right)$. After finding the coverage area of each aerial BS, we can find the maximum number of covered user points within each subarea by solving the following problem

$$
\begin{aligned}
& \underset{x_{c k}, y_{c k}, u_{i}}{\operatorname{maximize}} \sum_{i \in \mathcal{M}} u_{i} \\
& \text { subject to } \\
& \left(x_{i}-x_{c k}\right)^{2}+\left(y_{i}-y_{c k}\right)^{2} \leq R_{k}^{2}+M\left(1-u_{i}\right) \\
& y_{c k}-a_{k l} x_{c k}-b_{k l}+\frac{R_{k}}{\cos \left(\left|a_{k l}\right|\right)} \leq 0, \\
& \text { if } m_{k y}-a_{k l} m_{k x}-b_{k l} \leq 0 \\
& y_{c k}-a_{k l} x_{c k}-b_{k l}-\frac{R_{k}}{\cos \left(\left|a_{k l}\right|\right)} \geq 0 \\
& \text { if } m_{k y}-a_{k l} m_{k x}-b_{k l} \geq 0 \\
& u_{i} \in\{0,1\}, \forall i \in \mathcal{M} \\
& k=1,2, \ldots, K, l=1,2, \ldots, \mathrm{N}_{k l}
\end{aligned}
$$

The above optimization problem is a MINLP problem without non-convex constraints and can be easily sovled.

\section{SIMULATION RESULTS AND ANALYSIS}

In this section, we test the proposed techniques for user points distributed following Inhomogeneous Poisson process (IPP) with density function $\lambda(x, y)=\lambda_{c}\left(x^{2}+\right.$ $\left.y^{2}\right)$, where $\lambda_{c}$ is the average density of user points measured by users $/ \mathrm{km}^{2}$. An urban environment is considered. We assume $a=9.61, b=0.16, \eta_{\mathrm{LoS}}=1$, $\eta_{\mathrm{NLoS}}=20, f_{c}=2.5 \mathrm{GHz}$ and the optimal elevation angle $\theta_{\mathrm{opt}}=42.44^{\circ}$. Therefore, the radius $R$ corresponds to a path loss threshold $\gamma_{t h}=100 \mathrm{~dB}$ is calculated as $R=707 \mathrm{~m}$. To evaluate the benefit of the proposed algorithms, numerical results based on Monte Carlo simulations of the proposed LA and CL techniques are compared with the performance of CPT which serves as the benchmark. Note that due to the exponential increasing computational complexity of LA, the maximum $K$ value we use for the LA algorithm is four. The horizontal center of all deployed UAVs must fall inside the target area, and we assume the coverage areas outside the target area will not cause further interference to users outside the interested region. The benchmark CPT simply places circles with same size in a way that maximum coverage is achieved and 


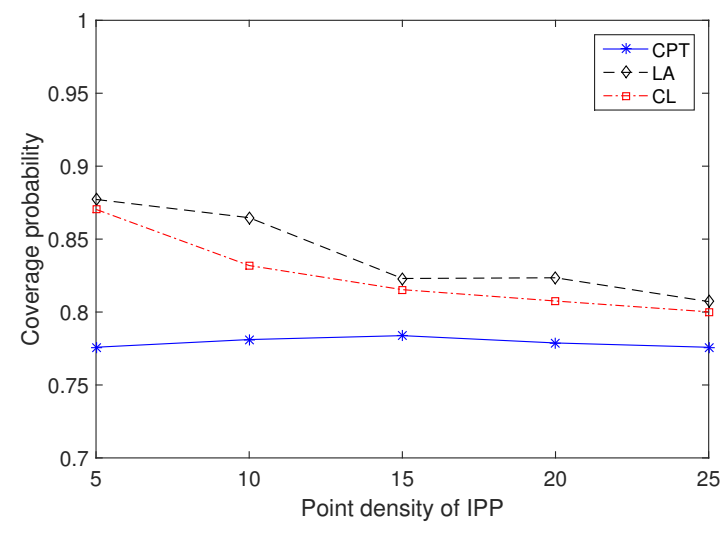

Fig. 4. User-coverage probability for users following IPP

none of these circles overlap. For CPT, the number of circles to be placed $N_{c p}$ only depends on the size of target area.

To illustrate the function of the proposed solutions, example UAV placement distributions are shown based on simulation for CPT, LA, and CL in Fig. 3, assuming $L_{s}=3 \mathrm{~km}$. Intuitively, when LA method is utilized, the placement of the new aerial BS always aims to cover most remaining user points in the target area, but the performance can be restricted by the previously deployed UAVs. In addition, The CL method aims to find the clustering properties among user points and is more robust to the change of user distributions than CPT.

For a fair comparison of the achievable coverage probability, a target area with $L_{s}=4 R$ is assumed, within which all the three methods can horizontally deploy at most four aerial BSs. The coverage probability of the three methods with a variable $\lambda_{c}$ is shown in Fig. 4. As can be seen, the proposed techniques achieve higher coverage probability than CPT. The performance of CPT is not affected by the density of users when a specific target area and density function is given, as it always places circles in fixed locations. On the contrary, our proposed algorithms are not restricted to fixed locations, but instead can be flexibly placed according to the change of user distribution and thus a performance gain is achieved.

In real scenarios, we may only have a limited number of UAVs for deployment. Therefore, it is meaningful to examine the coverage probability of the proposed techniques versus the number of available aerial BSs. We assume $L_{s}=3 \mathrm{~km}$ and the corresponding result is shown in Fig. 5. As expected, it can be seen that the proposed LA technique significantly outperforms other techniques since the UAVs are always deployed to cover a maximum number of remaining user points. When users are distributed following IPP, CPT outperforms the proposed CL method when only a small number of UAVs are available. In this case, a similar number of UAVs

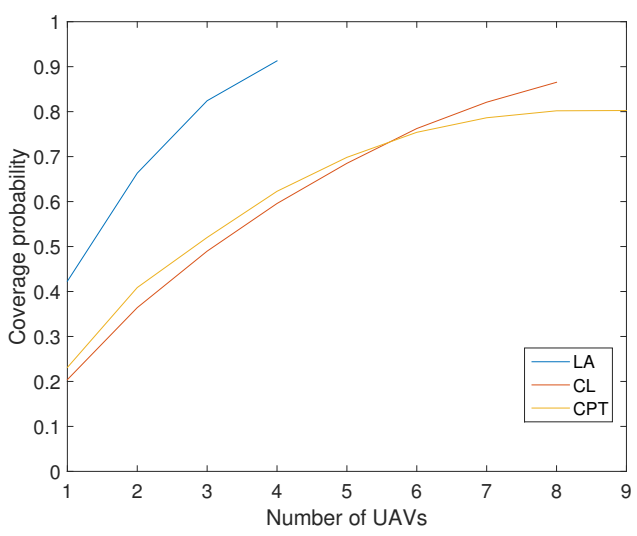

Fig. 5. User-coverage probability versus number of UAVs deployed

are deployed in a more tight way than the CPT, causing reduced coverage areas of certain aerial BSs and hence a smaller number of covered users within these areas.

\section{Conclusion}

In this paper, efficient deployment of multiple aerial BSs for maximizing the number of covered users while avoiding ICI is studied. A successive deployment method with linear approxiamtion and a simultaneous deployment method based on $K$-means clustering are proposed. Simulation results show that improved coverage probability is achieved by the proposed techniques when users are distributed unevenly.

\section{REFERENCES}

[1] Y. Zeng, R. Zhang, and T. J. Lim, "Wireless Communications with Unmanned Aerial Vehicles: Opportunities and Challenges," IEEE Communications Magazine, vol. 54, no. 5, pp. 36-42, May 2016.

[2] A. Al-Hourani, S. Kandeepan, and S. Lardner, "Optimal LAP Altitude for Maximum Coverage," IEEE Wireless Communications Letters, vol. 3, no. 6, pp. 569-572, Dec 2014.

[3] M. Alzenad, A. El-Keyi, F. Lagum, and H. Yanikomeroglu, "3-D Placement of an Unmanned Aerial Vehicle Base Station (UAV-BS) for Energy-Efficient Maximal Coverage," IEEE Wireless Coтmиnications Letters, vol. 6, no. 4, pp. 434-437, Aug 2017.

[4] M. Alzenad, A. El-Keyi, and H. Yanikomeroglu, "3-D Placement of an Unmanned Aerial Vehicle Base Station for Maximum Coverage of Users With Different QoS Requirements," IEEE Wireless Communications Letters, vol. 7, no. 1, pp. 38-41, Feb 2018.

[5] R. I. Bor-Yaliniz, A. El-Keyi, and H. Yanikomeroglu, "Efficient 3-D Placement of an Aerial Base Station in Next Generation Cellular networks," in 2016 IEEE International Conference on Communications (ICC), May 2016, pp. 1-5.

[6] M. Mozaffari, W. Saad, M. Bennis, and M. Debbah, "Drone Small Cells in the Clouds: Design, Deployment and Performance Analysis," in 2015 IEEE Global Communications Conference (GLOBECOM), Dec 2015, pp. 1-6.

[7] _ , "Efficient Deployment of Multiple Unmanned Aerial Vehicles for Optimal Wireless Coverage," IEEE Communications Letters, vol. 20, no. 8, pp. 1647-1650, Aug 2016.

[8] J. Lyu, Y. Zeng, R. Zhang, and T. J. Lim, "Placement Optimization of UAV-Mounted Mobile Base Stations," IEEE Communications Letters, vol. 21, no. 3, pp. 604-607, March 2017.

[9] R. Xu and D. Wunsch, Clustering. Wiley-IEEE Press, 2009. 